Journal of

Accident and

Emergency

Medicine 1994

$11,149-153$

\title{
Analysis of emergency department interpretation of electrocardiograms
}

\author{
E.R. SNOEY, ${ }^{1,3}$ \\ B. HOUSSET, ${ }^{1}$ P. GUYON, ${ }^{2}$ \\ S. ELHADDAD, ${ }^{2}$ J.VALTY ${ }^{2} \&$ \\ P. HERICORD ${ }^{1}$
}

${ }^{1}$ Department of Emergency Medicine and 2Department of Cardiology, Hospital Saint-Antoine, 184, rue du Faubourg Saint-Antoine, Paris, France and ${ }^{3}$ Department of Emergency Medicine, Highland General Hospital, 1411 East $31^{\text {st }}$ Street, Oakland, California

\section{SUMMARY}

The objective of the study was to determine the concordance of emergency physicians' and cardiologists' interpretations of emergency department (ED) electrocardiograms (ECG), to evaluate the impact of ECG misinterpretation on patient management, and to determine error rates as a function of the level of physician training and the specific ECG diagnoses. ECG interpretations were registered prospectively using a programmed-response data sheet. A second blinded interpretation by a staff cardiologist was assumed to be correct. Only ECG discrepancies with potential or probable clinical importance were considered as errors. The ED management of patients with ECG misinterpretations was reviewed by the investigators. The study was performed at an urban university hospital using 300 consecutive ED ECGs. The analysis found 154 errors of interpretation of which nine had probable clinical significance, and 56 had indeterminant significance. The concordance was weak at 0.69 (Kappa $=0.32$, weighted Kappa $=0.30)$ with a significant discordance (McNemar Chi 2:P<0.05). Error rates did not differ significantly between the diverse categories of physicians. In two cases, interpretation errors impacted patient management decisions but not patient outcomes. The most frequent errors involved repolarization abnormalities, ventricular hypertrophy and hemi-blocks. While discordance was significant, errors in ECG interpretation rarely impacted patient management. Prospective evaluation of ECG interpretation may be a useful means of gauging physician skills. It can also serve to focus educational activities on problem areas in electrocardiography.

Key words: Electrocardiogram, emergency department, interpretation

\section{INTRODUCTION}

The ECG is an important element in the evaluation of a wide range of ED pathology. The ED physician often must interpret the ECG in difficult circumstances: quickly, in isolation, without prior studies for comparison or a cardiologist's second opinion. Inaccuracy in the interpretation of the ECG by emergency physicians may result in inappropriate management decisions regarding patient treatment or disposition.

Several large studies reviewing missed acute myocardial infarction (AMI) in the ED have suggested that failure to interpret the ECG accurately was a important factor in patient management errors. ${ }^{1,2}$ Two recent studies found a significant discordance in the interpretation of ECGs between emergency medicine residents and cardiologists. ${ }^{3,4}$ Both studies were retrospective, comparing open-ended ED interpretations with a follow-up interpretation by a cardiologist. This methodology is strongly oriented towards a comparison of diagnostic accuracy while ignoring a more fundamental evaluation of ECG interpretation skills. A prospective study format permits a direct comparison of all elements of the ECG interpretation process without the population bias inherent in retrospective studies.

Despite ECG misinterpretation rates in the ED of up to $58 \%$, previous studies have found little clinical impact on patient management decisions. Although, based upon retrospective analyses, these results have raised questions regarding the relevance of quality assurance review of ECG interpretation. Assuming that ED practice standards, management strategies and patient populations may differ in France, the authors sought to prospectively determine the concordance of emergency physicians and cardiologists in the interpretation 
of ED ECGs, and to measure the clinical impact of interpretation errors.

\section{MATERIALS AND METHODS}

The institution is an urban, university-based teaching hospital located in central Paris. The ED is licensed to provide all levels of care and treats approximately 30000 adult patients per year. The department is staffed 24-h a day by first year post-graduate physicians, with a rotating pool of house officers assuming the night duty. No house officer has specific emergency medicine training. Attending physician supervision is provided during day time and evening hours only.

During a 2-month period, all patients, regardless of their ultimate disposition, for whom an ED ECG was performed were, included in the study. The most senior physician participating in the patient care was asked to complete a formal interpretation of the ECG using a programmed response data collection sheet. Each of the principal elements of the ECG interpretation (rate, rhythm, axis, QRS, hypertrophy and repolarization abnormalities) required a response ranging from normal to one or more of a series of potential findings. A synthesis of the findings with a probable diagnosis was registered at the end. A second blinded interpretation using an identical data sheet was performed by a staff cardiologist. This interpretation was assumed to be correct.

ED and cardiologist interpretations were compared. A misinterpretation was defined as any discrepancy between the cardiologist and the ED physician. The ECGs were subsequently classified by the investigators into one of three categories based on the potential clinical importance of the discrepancies: Category I - ECGs with no discrepancy or with an error of little potential importance, Category II - ECGs of indeterminate clinical importance, and Category III - ECGs with probable clinical importance (Table 1). These groupings define the potential for a specific ECG finding to change ED management (treatment, disposition etc.) if misinterpreted. In the situation where more than one discrepancy was identified, the most significant error defined the category designation.

For Category III patients (probable clinical importance), the ED charts were reviewed for evidence that ECG interpretations errors may have impacted patient management decisions. Patient outcomes were reviewed at the time of hospital discharge or within 10 days of discharge from the ED.
Table 1. Classification of ECG interpretation discrepancies

Category I: No discrepancy or ECG with minor discrepancy

Sinus tachycardia

Sinus bradycardia

Premature ventricular contractions

Premature atrial contractions

First-degree atrial-ventricular block

Axis abnormality

Atrial enlargement

Non-specific intraventricular conduction defect

Non-specific ST-T wave changes

Cateçory II: ECG abnormalites with indeterminant clinical significance

Right bundle branch block

Left bundle branch block

Left ventricular hypertrophy

Right ventricular hypertrophy

Left anterior hemi-block

Left posterior hemi-block

ST abnormality

Repolarization abnormality

Poor R wave progression

$Q$ wave

Category III: ECG abnormalites with probable clinical significance

Ischemia

Acute myocardial infarction

Prolonged Q-T interval

Wolf-Parkinson-White syndrome

Abnormal rhythm

Atrial fibrillation

Atrial flutter

Junctional rhythm

Pacemaker

Overall concordance of interpretations was determined using the $\mathrm{Chi}^{2} \mathrm{McNemar}$ test and Kappa test. Error rates based on the level of physician training were compared using the $\mathrm{Chi}^{2}$ test.

\section{RESULTS}

A total of 300 consecutive ECG interpretations were analysed, of which 102 were ultimately read as normal and 198 abnormal. The analysis found 154 $(51 \%)$ errors of interpretation of which $56(19 \%)$ were of little clinical significance (Category I), 89 $(30 \%)$ were of indeterminate significance (Category 
Emergency

department ECG

interpretation
II) and $9(3 \%)$ were of probable clinical significance (Category III).

If the analysis is limited to indeterminate or important errors (Categories II and III) only, one finds a total of 98 interpretation discrepancies. In 61 cases, the discrepant interpretation was the result of a missed finding by the ED (false negative). In 37 cases, the errors related to an overread by the ED not confirmed by the cardiologist (false positive). All but two of the nine Category III patients were hospitalized at the time of presentation.

The overall misinterpretation rate for all three categories was $51 \%$. If the analysis is limited only to those errors in Categories II and III (indeterminate or probable clinical significance), the rate improves to $32.7 \%$ with a Kappa $=0.32$, and weighted Kappa $=0.30$. The discordance was significant $(P<0.05)$. No significant difference in error rates was found between the various levels of physician training nor a consistent pattern of errors for a particular physician. The concordance rates for the ten most commons errors are listed in Table 2.

A total of 12 patients $(4 \%)$ in our study exhibited ECG evidence of AMI. All were identified correctly by the ED physician. By protocol, all patients had a cardiology consultation prior to initiating thrombolytic therapy.

$E D$ records were reviewed for each of the nine patients with Category III ECG errors. In two cases, interpretation errors may have impacted patient management decisions. The first case concerned a 76-year-old female who presented with weakness and near syncope. The ED interpreted her ECG as non-specific S-T segment abnormalities. The patient was given a diagnosis of dehydration and admitted

Table 2. Ten most common ECG elements with concordance results: Kappa Coefficient varies from 0 (no concordance) to 1.0 (maximum concordance)

\begin{tabular}{ll}
\hline ECG Finding & Kappa coefficient (0-1.0) \\
\hline Left ventricular hypertrophy & 0.41 \\
Left anterior hemi-block & 0.43 \\
ST segment abnormality & 0.45 \\
Bundle branch block & 0.54 \\
Right ventricular hypertrophy & 0.59 \\
Pathologic Q-wave & 0.64 \\
Left atrial enlargement & 0.72 \\
Premature atrial contraction & 0.92 \\
Premature ventricular & 0.92 \\
$\quad$ contraction & \\
Heart rate & 0.96 \\
\hline
\end{tabular}

to a ward bed for intravenous rehydration. The cardiologist reported a short P-R interval with repolarization changes characteristic of WolfParkinson-White syndrome. The authors believe this patient merited cardiac monitoring and a cardiology evaluation, given the context of a near syncopal event. She was discharged 2 days later and was well at a 1-week follow-up by telephone.

A second case involved a 66-year-old male hospitalized for an exacerbation of chronic obstructive pulmonary disease. Initial ED interpretation of the ECG suggested a left anterior superior hemi-block. The cardiologist reading noted S-T segment changes suggestive of anterolateral ischaemia. The patient was admitted to a ward bed for standard bronchodilator therapy. The ischaemic changes were recognized by the admitting team and the patient was ruled-out for myocardial infarction. The patient was discharged in good condition 7 days later with cardiology follow-up. The authors felt that admission to a monitored unit with cautious use of bronchodilator therapy would have been more appropriate give the initial ECG findings.

Using a review of hospital discharge summaries together with telephone follow-up for those discharged directly from the ED, it was determined that none of the nine Category III patients experienced a near-term complication.

\section{DISCUSSION}

The analysis confirms the significant discordance between ED and cardiologist interpretations of ECGs previously described. The importance of accurate ECG interpretation has been brought into greater focus recently with the more routine use of thrombolytics in the ED and the unmitigating medicol-legal environment. Time to thrombolytic therapy has been confirmed as an independent variable in improving survival for patients with AMI. As such, a timely initiation of thrombolytic therapy may preclude a concurrent reading of an ECG by a cardiologist. Several studies have evaluated emergency physician accuracy in identifying patient candidates for thrombolytic therapy. In a study by Ho et al., 13 out of 236 patients with ECGs indicative of acute myocardial infarction (AMI) were missed by emergency physicians. ${ }^{1}$ Mistakes most often involved small S-T segment abnormalities or bundle branch blocks. A second study by Lee et al. found that 21 out of 445 ECGs indicating AMI had been misinterpreted by emergency physicians. ${ }^{2}$ In a related study population of patients with missed 
AMI, 16 of 34 ECGs (47\%) with evidence of AMI or ischaemia were interpreted as normal or showing nonspecific ST-T wave abnormalities. ${ }^{2,5}$ Other studies reviewing the problem of missed ED AMI and litigation have found ECG misinterpretation to be a contributing cause in up to $47 \%$ of cases. ${ }^{6,7}$ In the USA, missed AMI continues to occupy the number one position for compensation awarded in malpractice litigation. It comprises $10 \%$ of all cases that go to litigation and between 20 and $45 \%$ of all dollar losses in the field of emergency medicine. ${ }^{8}$

This current study addressed the issue of ECG interpretation in the setting of all forms of ED pathology without focusing specifically on ischaemic heart disease. In a recent retrospective study of ECG interpretation at an emergency medicine teaching programme, Kuhn et al. found error rates of $\mathbf{8 . 6}$ and $8.3 \%$ for ECGs with indeterminate and probable clinical importance respectively. ${ }^{3}$ In only three cases $(0.9 \%)$ was patient ED management felt to be affected. Westdorp et al. had similar results in a review of 716 ECGs in patients discharged from the ED. ${ }^{4}$ Errors rates of 40.6 and $17.5 \%$ were reported for indeterminate and probable errors respectively. ECG misinterpretation was judged to have changed patient care in two cases $(0.5 \%)$. While this study found a much smaller rate of errors with probable clinical importance $(3 \%)$, overall discordance of ECG interpretation remained significant. The most common errors involved the identification of repolarization abnormalities, bundle branch blocks and hemi-blocks. ED physicians were most adept at identifying premature contractions, axis abnormalities and rhythm disturbances. Of the nine potentially significant errors classified in Category III, six were represented by three diagnoses: prolonged Q-T syndrome, Wolf-Parkinson-White syndrome and atrial fibrillation (Table 3). In two cases, interpretation errors impacted management decisions but not patient outcomes.

The importance of ancillary findings such as intraventricular conduction disturbances and re-

Table 3. Category III ECG Diagnostic Errors

Wolf-Parkinson-White syndrome

Prolonged Q-T syndrome

Atrial fibrillation

Junctional tachycardia

Atrial flutter

Anterolateral ischaemia by S-T segment polarization abnormalities has been emphasized in a recent series of articles reviewing in-hospital complications from AMI. ${ }^{9,10}$ Bundle branch blocks and left ventricular hypertrophy, in addition to myocardial ischaemia, were found to be prognostic for in-hospital cardiac complications. The authors suggest that suspected AMI patients without these ECG findings (patients with normal, non-specific, or unchanged ED ECGs) may be safely admitted to an intermediate care unit. Cardiac care unit overload and hospital expense could thereby be reduced. Given the high misinterpretation rate for bundle branch blocks and left ventricular hypertrophy found in this and other studies, it is conceivable that the clinical relevance of these findings may change as patient admission protocols evolve. Because no intermediate cardiac care unit exists at our institution, no patient care was affected.

This study addresses not only the diagnostic accuracy of ED ECG interpretation, but also basic interpretation skills as defined by a direct comparison of each element of the ECG. While retrospective studies of ECG concordance provide useful quality assurance information in terms of patient outcomes, they are less precise in their ability to evaluate physician electrocardiographic skills or deficiencies. The evaluation of individual physicians or the orientation of educational activities in the domain of ECG interpretation may be well served by this kind of analysis.

Despite a significant overall discordance, ED interpretation errors rarely affect patient management decisions. Kuhn et al. argue that systematic review of ED ECG interpretation by a staff cardiologist is not indicated based on these findings. ${ }^{3}$ Clearly, if a quality assurance activity is to be judged according to its impact on patient management or outcome, the effort involved in a systematic review of all tracings seems unwarranted. However, ED management decisions depend on a host of different factors of which the ECG is but one. Retrospective review of patient management and outcome is therefore likely to be an insensitive means of determining the clinical relevance of an ECG interpretation error. If one's goal is to identify ED management mistakes or poor patient outcomes, other quality assurance strategies may prove more effective, such as callback of predetermined high-risk patients.

There were several potential limitations to this study. The ECG 'gold standard' used was the interpretation of one staff cardiologist. We did not develop a consensus process to review interpretation discrepancies. The evaluation of patient 
Emergency

department ECG

interpretation management, based on a retrospective review of patient charts and clinical outcomes, was limited to Category III patients only. It was, however, performed on a consensus basis between two experienced ED physicians. Lastly, the author's goal was to evaluate physician accuracy in ECG interpretation. The impact of using pre-programmed data sheets on the quality of ED ECG interpretations was not tested a priori. It is likely that physicians in this study were more fastidious in their interpretations given the prospective study format. This is supported by the relatively low error rate in comparison to previous studies of this kind.

\section{CONCLUSION}

This analysis found a significant discordance between ED physicians and cardiologists in the interpretation of ED ECGs. Despite this discordance, errors were deemed unlikely to impact patient management decisions. The authors of this study support periodic prospective reviews of ECG interpretation skills as a means of evaluating overall ED performance and individual physician competence. The ECG remains one of the most common, useful and cost effective tests available to the ED physician. The skillful interpretation of the ECG should continue to be a focus of quality assurance and educational activity.

\section{REFERENCES}

1. Ho M., Kudenchuk P., Eisenberg M. et al. (1990) Patient selection for thrombolytic therapy: Emergency physicians versus electrocardiographer (abstract).
Journal of the American College of Cardiology 15, $192 A$.

2. Lee T., Weisberg M., Brand D. et al. (1987) Candidates for thrombolysis among emergency room patients with acute chest pain. Annals of Internal Medicine 110, 957-962.

3. Kuhn M., Morgan M. \& Hoffman J. (1992) Quality assurance in the emergency department: Evaluation of the ECG review process. Annals of Emergency Medicine 21, 10-15.

4. Westdorp E., Gratton M. \& Watson W. (1992) Emergency department interpretation of electrocardiograms. Annals of Emergency Medicine 21, 541-544.

5. Lee T., Rouan G. \& Weisberg M. (1987) Clinical oै Characteristics and natural history of patients with $\vec{\circ}$ acute myocardial infarction sent home from the emergency room. American Journal of Cardiology 60, 219-224.

6. Karcz A., Holbrook J., Auerbach B. et al. (1990) Preventability of malpractice claims in emergency medicine; A closed claims study. Annals of Emergency Medicine 19, 865-873.

7. Rusnak R., Stair T., Hansen K. et al. (1989) Litigation against emergency physicians: Common features in cases of missed myocardial infarction. Annals of Emergency Medicine, 1029-1034.

8. Hill M. (1989) Failure to diagnose myocardial infarction: The emergency physician's greatest liability in practice. QRC Advisor 6, 1-8.

9. Stark M. \& Vacek J. (1987) The initial electrocardiogram during admission for myocardial infarction. Archives of Internal Medicine, 843-846.

10. Villanueva F., Sabia P., Afrookteh A., Pollock S., Hwang L. \& Kaul S. (1992) Value and limitations of current methods of evaluating patients presenting to the emergency room with cardiac-related symptoms for determining long-term prognosis. American Journal of Cardiology 69, 746-750. 